\title{
Integralidad de la gestión desde la transdisciplinariedad ${ }^{*}$
}

\section{Integrity Management from transdisciplinarity}

Recibido: 22 de junio de 2014

Revisado: 14 de septiembre de 2014

Aceptado: 13 de febrero de 2015

Néstor Daniel Valois Cubillos**

Consultor Independiente

\section{RESUMEN}

El presente artículo describe los principales aspectos para construir y probar una metodología para la integralidad de la gestión en las organizaciones. Se incluyen siete modelos de gestión normalizados, metodología que trasciende la combinación de modelos de gestión al considerar a las organizaciones como un sistema y no a la organización con varios sistemas. Se fundamenta en la transdisciplinariedad 0 acción transversal mediante el ciclo "desorden, organización, orden, interacción". Considera aspectos del entorno e internos, prioriza necesidades y propósitos, ordena su implantación y somete el sistema a interacciones.
La investigación parte de la consulta a expertos en cada modelo de gestión, explora las metodologías existentes para integrar sistemas de gestión, toma la transdisciplinariedad mediante la reflexión todo-parte: entorno-empresa (sistema), relaciona los requisitos de cada norma con los siete saberes necesarios para la educación del futuro y como resultados se obtienen el modelo de gestión y la metodología para la integralidad. La metodología se aplica y luego se concluye que permite incrementar la probabilidad de conseguir los objetivos empresariales y hacer viable a la empresa como sistema.

\footnotetext{
* Artículo de investigación.

** Ingeniero Civil. Especialista en Análisis de Datos. Especialista en Gestión Ambiental. Magíster en Calidad y Gestión Integral. Correos electrónicos: davalois14@gmail.com y davalois14@yahoo.com
} 
La metodología es aplicable en organizaciones públicas o privadas que no solo requieren cumplir normas, sino que deben ser sostenibles en el tiempo, con influencias transformadoras como pensamiento global, cambio, finitud de los recursos naturales, incertidumbre, productividad e información virtual.

Palabras clave: complejidad, gestión integral, sistema de gestión, transdisciplinariedad.

\section{ABSTRACT}

This article describes the main aspects to build and test a methodology for the comprehensive management in organizations. Seven models of standardized management methodology that transcends the combination of management models to consider the company as a system and not the company with several systems are included. It is based on transdisciplinary or transverse action by the cycle: Disorder, Organization, Order, Interaction. It considers environment and internal aspects, priority needs and purposes, ordering its implementation and submits the system to interactions.

The research begins consulting experts in each management model. Explore existing methodologies to integrate, making transdisciplinarity by reflection All - Part, Environment - Enterprise (system). Relate the requirements of each standard with the seven knowledges for the future education and as a result the management model and methodology for integrity is obtained. The methodology is applied and concludes that allows to increase the probability of achieving business goals and makes feasible the company as a system.

The methodology is applicable in public or private organizations that require not only meet standards but must be sustainable over time, transforming influences as global thinking, change, finiteness of natural resources, uncertainty, productivity and virtual information.

Keywords: Complexity, integrated management, management system, transdisciplinarity.

\section{INTRODUCCIÓN}

El ejercicio de la gestión empresarial presenta un reto fundamental al considerar la presencia de restricciones normativas y legales, los condicionamientos y la dinámica de mercado, los cambios, el efecto de la globalización, la cultura organizacional y la competitividad, todo ello en medio de la necesidad de sostenibilidad en el tiempo y adaptación al cambio. Los modelos de gestión normalizados han procurado instrumentos para mitigar los impactos no deseados, producto del estado anteriormente señalado. Sin embargo, la diversidad de normas por implementar vuelve compleja la intervención y probablemente más ineficiente que alguna otra metodología que permita convergencia y unicidad en la gestión nuclear o fundamental de las organizaciones. Concebir la organización como una estructura piramidal — como consecuencia de las premisas de especialización, división en áreas y destrezas en la individualidad - dificulta la introducción de estos modelos de gestión, del enfoque por procesos y, con mayor razón, de la concepción de la organización como sistema. La metodología para la integralidad ${ }^{1}$ en la gestión es planteada como una alternativa que da respuesta a la problemática de implementar varios sistemas en una organización.

1 Esta investigación diferencia entre gestión integral e integralidad de la gestión. La primera combina requisitos y se realiza a partir de los modelos de gestión normalizados existentes. La segunda parte del entorno, las necesidades del sistema o de la empresa, la cultura, el modelo de integralidad, la transdisciplinariedad e involucra las intencionalidades $\mathrm{y} / 0$ instrumentos al servicio del objetivo de la sostenibilidad por medio de la transformación continua. 
Trasciende la suma o combinación de requisitos originados en las normas de gestión disponibles, es decir, la elaboración de tablas de equivalencia entre requisitos de las normas. En este sentido, la estructura de alto nivel emitida por la Organización Internacional de Normalización (ISO) facilita la unicidad en el modelo de gestión y envía una señal más concreta para el enfoque por procesos.

Es importante considerar los esfuerzos, con enfoque disciplinario individual, que se han realizado en pro de facilitar la gestión en las empresas; muestras de ello, en general, son las normas técnicas ISO 9001 (ICONTEC, 2008) sobre el sistema de gestión de la calidad. Y en Colombia, la NTCGP1000 (ICONTEC, 2009), para la gestión de la calidad en las entidades del Estado; la norma OHSAS 18001 (ICONTEC, 2007), para el sistema de gestión de la seguridad y la salud ocupacional; el Modelo Estándar de Control Interno - MECI (DAFP, 2014); la norma ISO 31000 (ICONTEC, 2011), sobre gestión del riesgo; la norma ISO 27001 (ICONTEC, 2013), que aborda la seguridad de la información; la guía ISO 26000 (ICONTEC, 2010), para la responsabilidad social; y la norma ISO 14001 (ICONTEC, 2004), para la gestión ambiental. Otros esfuerzos los podemos observar con la emisión de las normas PAS 99 (BSI, 2012) y UNE 66177 (AENOR, 2008), para facilitar la integración de los sistemas de gestión, e incluso en el ejercicio de las auditorías de gestión con la norma ISO19011 (ICONTEC, 2012), que facilita las auditorías combinadas. En Colombia se destacan el surgimiento de la norma NTCGP1000, en su versión 2009, que combina lineamientos de la gestión de la calidad y del Modelo Estándar de Control Interno - MECI, así como el Decreto 2482 de 2012, que proyecta el Modelo Integrado de Planeación y Gestión. Igualmente, sobresale la estructura de alto nivel emitida por la International Organization for Standarization (ISO), AnnexSL, entre otros muchos esfuerzos por generar una integración entre los diversos sistemas de gestión.

La forma de gestionar las empresas se ha modificado desde los paradigmas de Fayol y Weber - en cuanto a la especialización del trabajo y la concepción de una estructura organizacional jerárquica-, hasta los modelos de gestión normalizados basados en el concepto de sistema. En todo caso, en las empresas subyacen los paradigmas mencionados y se amalgaman con el enfoque de procesos, en ocasiones creando distancias entre la gestión y el modelo configurado, esto es, que los resultados y las rutinas no se relacionan con el modelo de gestión, generando desperdicios, contraórdenes o simplemente una percepción inadecuada de la contribución de los sistemas de gestión al logro de los objetivos organizacionales. Estas características se constituyen en limitaciones para la gestión con base en la integralidad. El lenguaje propio de la complejidad y la cultura organizacional se suman a las limitaciones observadas. El modelo para la integralidad de la gestión las tiene en cuenta, y mediante su eje articulador minimiza los efectos de dichas limitaciones.

Afrontar el concepto de sistema implica predeterminar el enfoque del fundamento epistemológico. Así, la ciencia clásica no es perfectamente adecuada, según lo plantea Bertalanffy (1976). También Ruy Pérez (1990), con su frase "en dónde se ha equivocado la ciencia", contiene una indignada protesta en contra de los filósofos y científicos. El paradigma de la complejidad planteado por Morin (1997) presenta el concepto de sistema, al cual le asigna las características de desorden, organización, orden e interacción, y otros estudios sobre la complejidad desde el siglo XX proponen integrar a ellas la ciencia y la filosofía (Rodríguez, 2011). La presente investigación toma esas características, las identifica para hacer su aplicación a las organizaciones y propone que estas características se modelen en secuencia. Por desorden debería entenderse los 
momentos de cambio en la empresa; por organización, el momento en que se establecen criterios para establecer prioridades; por orden, todo lo relacionado con la planificación de acciones, recursos y plazos; y finalmente está la interacción, que incluye las acciones de todos los elementos de la empresa o sistema. Debe observarse que esta secuencia plantea diferencias con el ciclo tradicional de la gestión PHVA (planificar, hacer, verificar y actuar).

La metodología para la integralidad de la gestión se construye con la participación de siete expertos en siete modelos de gestión normalizados: Gestión de la Calidad, Gestión ambiental, Gestión en Seguridad y Salud ocupacional, Gestión del Riesgo, Modelo Estándar de Control Interno - MECI, Responsabilidad Social y Seguridad de la Información. Esta metodología se integra con los siete saberes propuestos por Morin (1999). Igualmente, sigue los lineamientos de transdisciplinariedad dados por el Proyecto Educativo Institucional (PEI) de la USTA (2004).

Para esta construcción, fue necesario considerar el estado del arte de los conceptos sistema, gestión, complejidad, modelos de gestión, integralidad e integral, aplicando la reflexión dialógica todo-parte, para soportar teóricamente el diseño del sistema.

En la primera etapa de la investigación, se realizó la consulta a expertos mediante un formulario semiestructurado, validado mediante prueba Cronbach, (Oviedo, 2005) y se emplearon talleres para identificar consensualmente cuál de las metodologías aplicadas por los expertos es la más incluyente conforme a los siete modelos de gestión. Luego, la metodología identificada se enriqueció con la reflexión dialógica, el paradigma de la complejidad y el aporte de los siete saberes necesarios para la educación (Morin, 1999). La investigación proporciona adicionalmente un resultado en cuanto al mapa inacabado e incompleto de los conocimientos y las fronteras de los conocimientos identificados, construido con el fin de ubicar el concepto de sistema en la historia del conocimiento.

En la segunda etapa, la de investigación aplicada, se diagnosticó, mediante una encuesta, el nivel de implantación de los modelos de gestión en una empresa seleccionada. Para lograr el diagnóstico, se elaboró y validó un instrumento que correlaciona los siete saberes propuestos por Edgar Morin, con los requisitos normativos de los siete modelos de gestión, traducidos en conductas esperadas o cultura organizacional observable, como plantea Soracipa (2009). La investigación incluye la determinación de conductas reflejo de la cultura existente en estados que van desde no practicar la conducta examinada, hasta interiorizarla (capacidad de enseñar mediante el ejemplo). Igualmente, se realizó un taller en grupo con los trabajadores del proceso misional y se trianguló la información con autoevaluación, heteroevaluación y los datos anteriormente obtenidos, "el aprendizaje resulta de la interacción con la sociedad" (Vigostky, 1986).

Con el resultado del trabajo, se definieron un modelo y una metodología que permiten integrar, en una gestión global y única, las diversas perspectivas de gestión en una organización, manteniendo su conexión con el entorno y estableciendo prioridades según los riesgos y las oportunidades.

\section{MÉTODOS}

Se adoptó un enfoque mixto tipo explicativo porque permite recolectar, analizar y vincular información de carácter cualitativo, cuantitativo, y ello se realizó en dos grandes fases. En la primera fase se identificaron las fuentes de información con los expertos en cada una de los siete modelos de gestión, de quienes se obtuvo no solo su conocimiento y nivel alcanzado, sino 
su experiencia por haber implementado como mínimo una vez un modelo de gestión en la práctica. Mediante prueba de Cronbach, se validó la opinión experta sobre el modelo de gestión más comprensivo, es decir, cuál modelo es más incluyente con fundamento en los requisitos.

De otra parte, se hizo una amplia revisión bibliográfica de conceptos vinculados a complejidad, organización, cultura y sistema, esto con el fin de conseguir un estado del arte, que se refleja en el mapa de conocimiento incompleto e inacabado.

Posteriormente se llevó a cabo la reflexión dialógica, que permitió desplegar las relaciones e interdependencias a partir de las preguntas del todo a la parte y de la parte al todo.

Se definió la hipótesis por probar: la metodología para integrar siete modelos de gestión es conveniente. El concepto de conveniencia se definió como la percepción que tienen los encuestados sobre la probabilidad en el logro de los objetivos de la entidad si se implementan los 7 modelos de gestión.

En la segunda fase también se diseñaron instrumentos para la recolección de datos desde diferentes perspectivas: encuesta, entrevista en profundidad, autoevaluación y heteroevaluación. Para el diseño de estos instrumentos, se elaboró una matriz que relacionara los siete modelos de gestión analizados con los siete saberes propuestos por Morin (1999).

El primer instrumento empleado fue la encuesta, que permitió obtener una visión pluralista (directivos, delegados de sistemas de gestión, y responsables de proceso) de la percepción que tienen estos diferentes actores dentro de la empresa sobre el nivel de implementación de los siete modelos de gestión. La encuesta preguntó el grado de implantación de cada uno de los siete modelos de gestión considerando una escala en la cual el puntaje se asignó de la siguiente forma: "En la organización": apenas se conoce = 1, se divulga frecuentemente $=2$, hay procedimientos formales $=3$, siempre se hace, está interiorizado $=4$ y se enseña sistemáticamente $=5$.

Los instrumentos de autoevaluación y heteroevaluación se construyeron con una tabla de doble entrada o matriz, para relacionar cada conocimiento con los requisitos de cada norma dirigidos a los participantes de los procesos misionales.

Otro instrumento utilizado fue la evaluación del procedimiento operativo, que fue realizada por los responsables del proceso y que consistió en identificar en el texto de dicho procedimiento, instrucciones concretas o específicas de los temas que se preguntaban en el instrumento y que coincidieran con los elementos confrontados en la matriz previamente elaborada. Se realizó un taller con la presentación de los siete saberes de Edgar Morin en el que se mostraron sus aplicaciones prácticas. Al finalizar el taller, se solicitó a los participantes una nueva autoevaluación, sabiendo que ya se les habían presentado los siete saberes. Cada ejercicio de evaluación determinó el nivel de apropiación de los saberes y su relación con la probabilidad de lograr los resultados de la gestión en la organización, relacionados con los productos, resultados económicos, frente al personal, el cumplimiento legal y los relacionados con la comunidad.

Otra visión obtenida fue gracias a la heteroevaluación, realizada por el jefe del proceso. Es decir, se quiso determinar cómo el jefe observa los comportamientos de las personas a cargo, que reflejan la cultura organizacional, en la práctica, y la aplicación de los requisitos de las normas.

El último instrumento empleado fue la entrevista en profundidad semiestructurada, con el fin de conocer el entorno cercano al proceso intervenido y su relación 
con la aplicación de los siete saberes, comparación tipo cualitativo que complementó las evaluaciones cuantitativas.

Finalmente se tabuló, trianguló y analizó la información para confirmar o negar la hipótesis planteada.

\section{RESULTADOS}

\section{Análisis con expertos}

Inicialmente se obtuvo la comprensión de cada modelo de sistema de gestión, es decir, cuál es más incluyente y cuál es su relación con los demás sistemas de gestión, por medio del análisis de expertos. Aquí, los expertos manifiestaron, desde su perspectiva, que sería muy complejo el intento de realizar la implementación conjunta de los siete modelos y opinaron que debería implementarse por etapas, dando prioridad al sistema de gestión de la calidad, y luego, sobre esta base, implementar otros. El coeficiente alfa de Cronbach a partir de 0,7 es bueno como criterio de validación (Oviedo y Arias Campo, 2005). Los resultados arrojaron un valor de $69,72 \%$, el cual indica que la confiabilidad es buena. De allí se concluye que el modelo MECI es el más comprensivo de los modelos evaluados por los expertos. Igualmente, se referenció al modelo PAS 99 como la metodología apropiada para la integración de los sistemas de gestión. Se debe recordar que dicha norma plantea la integración solo de los sistemas de gestión para la calidad, el medioambiente y la salud ocupacional; adicionalmente, escala la implementación conforme al nivel de implementación de los modelos, es decir, debe tenerse en cuenta el modelo que se está implementando para implementar luego otros modelos. El modelo propuesto para la integralidad de la gestión se centra en la gestión de la organización o sistema y no en los modelos con sus requisitos, priorizando las necesidades de la organización y no los requisitos que se deben cumplir de cada modelo, aunque los tiene en cuenta. Por otra parte, y con fundamento en los aportes de cada experto, se estableció que las etapas generales de implementación son las siguientes: compromiso, sensibilización, aplicación de autoevaluación inicial, planificación, determinación de riesgos, determinación de información y comunicación, realización de auditoría y planes de mejoramiento. Nótese que antes de determinar los objetivos, es paso obligado el diagnóstico referido a los contenidos del modelo. Igualmente, es importante señalar que en esta metodología no se pregunta sobre la cultura organizacional, no se indaga sobre responsabilidad social y no se establece un nivel de probabilidad del cumplimiento de objetivos organizacionales. El modelo para la integralidad de la gestión se fundamenta en la cultura organizacional en la medida que en el diagnóstico establece el nivel de implementación de los modelos en la práctica o vivencia individual y colectiva.

\section{Revisión bibliográfica}

De la revisión bibliográfica se obtuvo el mapa de conocimiento incompleto, inacabado e informe. Como tal, su importancia radica en la compilación de las corrientes de pensamiento centradas en el concepto de sistema. Desde el conocimiento fundado en doxa (opinión) hasta el positivismo, fue incorporado al análisis sobre el concepto de sistema, con base en autores que presentan diversas conceptualizaciones y puntos comunes frente al sistema (Bertalanffy, 1976), (Morin, 1984), (Pérez, 1990), (Schvarstein, 2003), (Rodríguez y Aguirre, 2011). Para la investigación, se identificó como pertinente la concepción de la organización como unidad social y, a la vez, como sistema social.

La figura 1 muestra la aproximación al mapa de conocimiento incompleto (no está todo el conocimiento), inacabado (no se deja de construir porque la ciencia es perfectible) e informe (porque las formas mostradas 
no necesariamente representan los límites del conocimiento), construido con el fin de señalar que el concepto de sistema comparte y excluye conocimientos. Por ejemplo, con la teoría general de sistemas comparte la particularidad de que los elementos son diversos y están interrelacionados en el sistema, mientras que un conjunto no es un sistema. Con las llamadas ciencias de la complejidad comparte el concepto de complejo y, a su vez, estas ciencias no lo integran por considerar que no hay aplicabilidad de sistemas en sociología. Las líneas discontinuas representan espacios del conocimiento que pueden compartirse 0 interactúan con otros, como conocimientos permeados. Finalmente, los modelos de gestión tienen en común con los sistemas, que se refieren a sistemas, aunque si la organización no se concibe como sistema, se comportará como áreas interrelacionadas o como sistemas de gestión combinados.

Como resultado de la anterior conceptualización, la metodología para la integralidad en la gestión incluye conocimiento global y conocimiento contenido en los modelos de gestión. Siempre que se considere la organización como sistema, es posible la identificación de las llamadas partes interesadas externas al sistema y en el interior de la organización, reflejadas en su cultura organizacional, como lo muestra la figura 2.

La organización como sistema se observa interactuando con su medio y consigo misma, de esta forma se aplica el concepto sistema en su plenitud.

Figura 1. Mapa de conocimiento inacabado

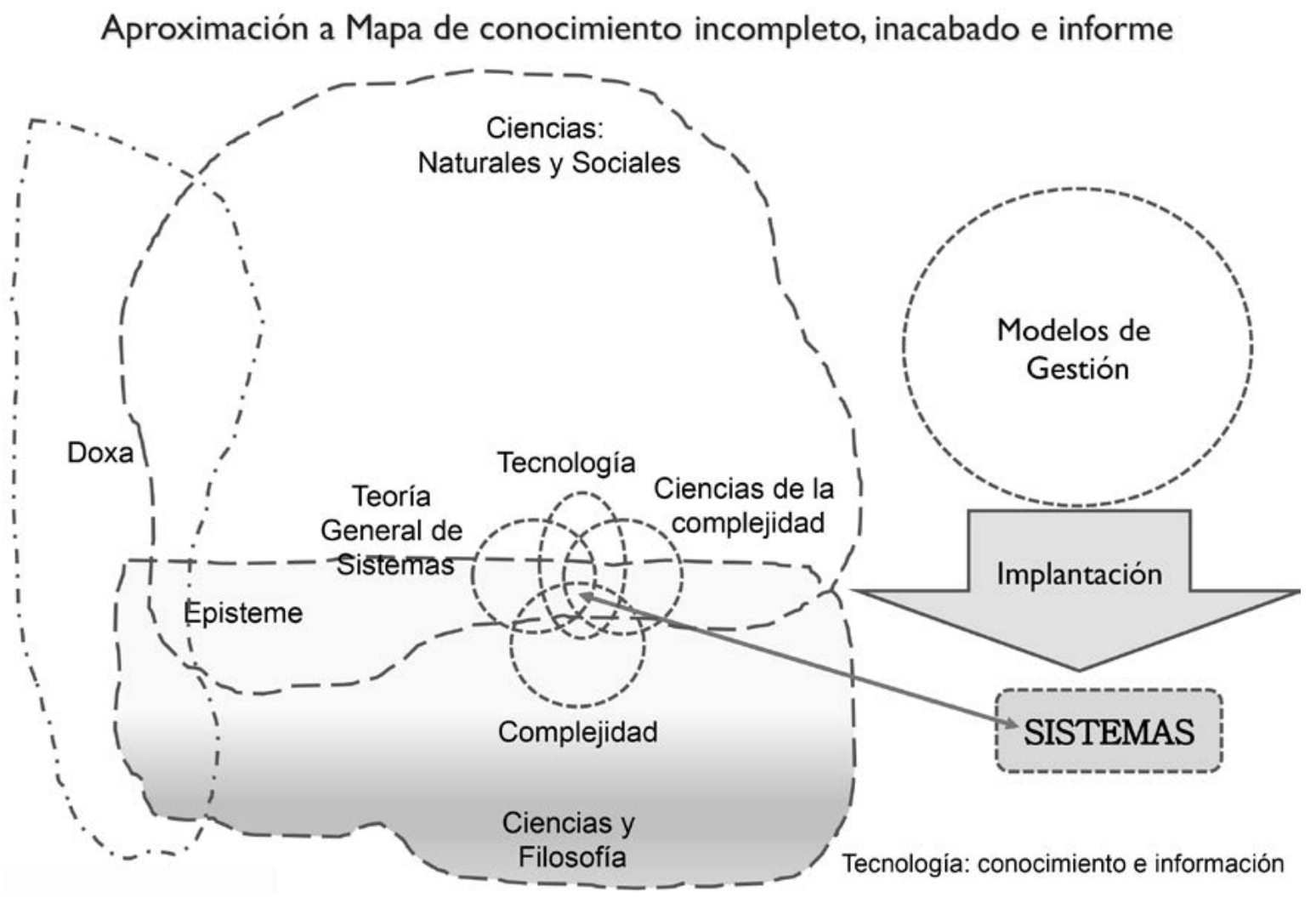

Fuente: construcción propia. 
Figura 2. Interacciones del conocimiento

\section{Interacciones del conocimiento}

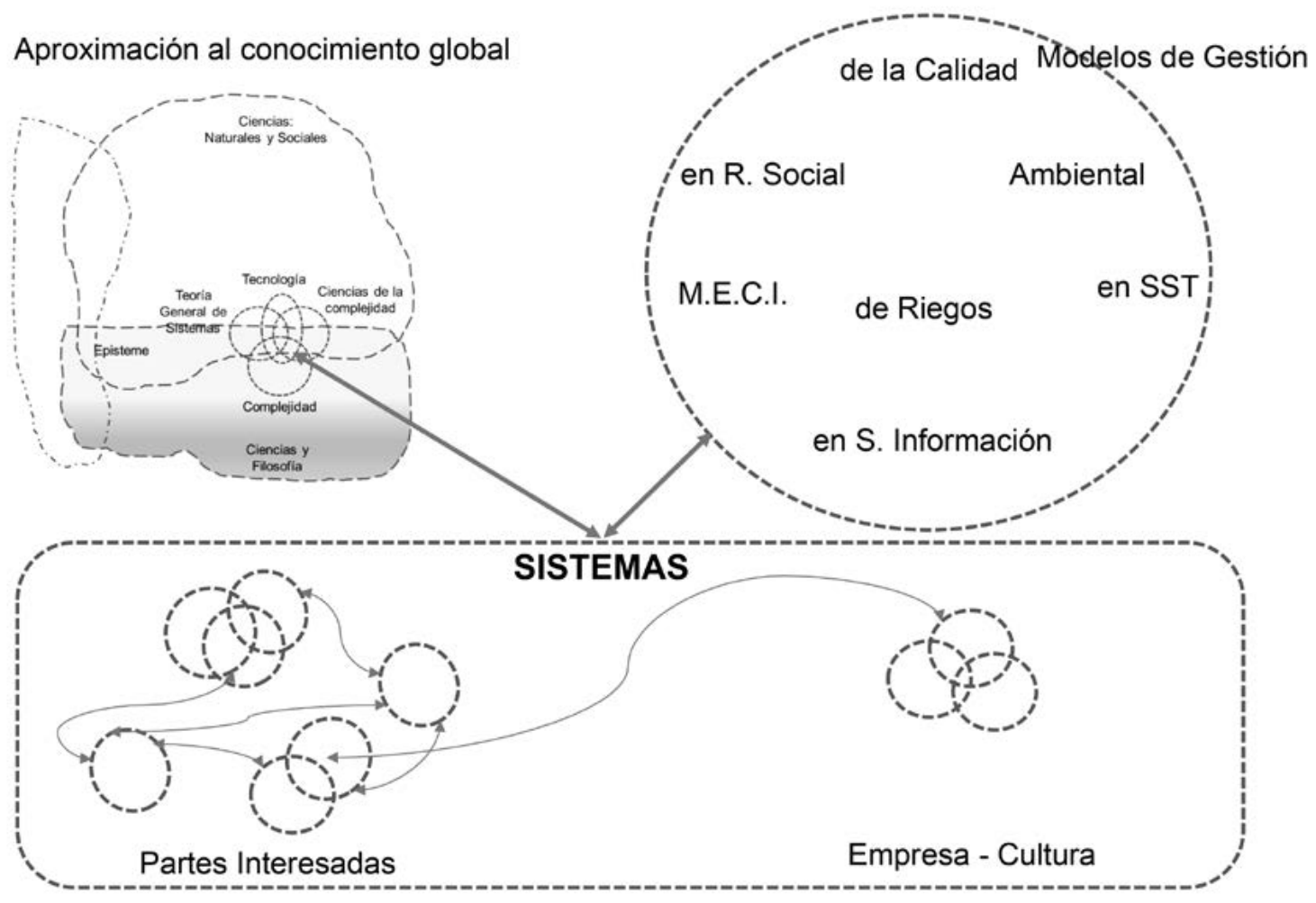

Fuente: construcción propia.

\section{Análisis reflexivo}

La reflexión dialógica es un ejercicio que permite establecer, mediante preguntas y respuestas, las relaciones entre el todo y la parte. Su aporte fundamental, en este caso, fue evitar el sesgo en la investigación, resultado propio del método cuando excluye variables que considera no pertinentes para el objetivo de la investigación, así como por la delimitación del alcance. La metodología para la integralidad de la gestión, aunque identifica objetivos y alcance, no se limita a ellos y complementa la información mediante esta reflexión dialógica planteada por Edgar Morin (1987): "Tengo por imposible concebir las partes al margen del conocimiento del todo, tanto como conocer el todo sin conocer particularmente las partes". Se describe a continuación un ejemplo de la aplicación de la reflexión dialógica en torno a los conceptos de sistemas, gestión y cultura en el desarrollo de esta investigación.

- Reflexión: El método científico forma parte de la súper especialización del conocimiento. ¿Qué lineamientos existen en la universidad Santo Tomás, en convenio con el ICONTEC, sobre la investigación?

- Respuesta: "La visión global —nunca definitiva一, renovadora del estudio general, será imposible si no se llega al nivel de discurso transdisciplinario, que hace emerger de la confrontación de las disciplinas, en una mirada unificadora necesaria para la comprensión holística. Para lograr esto, la 
confrontación interdisciplinaria no significa confrontación entre los investigadores...”. Es decir, la Universidad promueve el desarrollo de investigación transdisciplinaria (Universidad Santo Tomás, PEI, 2004).

- Reflexión: ¿Cuál será una aplicación adecuada en un proyecto de investigación?

- Respuesta: La que incluya los lineamientos de la USTA y los del ICONTEC.

- Reflexión: ¿Qué lineamientos provee el ICONTEC?

- Respuesta: "Visión. Lograr que los programas del convenio USTA-ICONTEC sean reconocidos a nivel nacional e internacional como generadores de conocimiento y como la mejor alternativa de formación en sistemas de gestión". Los sistemas y el pensamiento complejo de Edgar Morin son plenamente aplicables, imbricados, relacionados. Se concluye que integrar varios modelos con el paradigma de la complejidad es un buen propósito.

- Reflexión: ¿Cómo realizarla?

- Respuesta: Mediante las disposiciones contenidas en la Guía para elaboración del trabajo de grado. En dicha guía se referencia el libro de Leonardo Schvarstein (2003), La Inteligencia social de las organizaciones, en el cual se encuentra el concepto de organización caórdica. ¿Cómo aplicar el concepto de organización caórdica al proyecto?

- Respuesta: Los temas más controversiales frente a la tradición de empresa son la distribución del poder, la distribución de la autoridad y solo trabajo en equipo. Al respecto, corrientemente se circula por una ruta no común, porque lo arraigado es el establecimiento de la estructura organizacional como mecanismo para definir los cargos, la jerarquía, el conducto regular. Así se muestra en el caso de la organización propuesta por Fayol, en la que las divisiones, las áreas y, en general, la burocracia, están planteadas como formas de especialización en el trabajo. Por lo anterior, es mayor la coincidencia con el concepto de sistema entre una organización caórdica que una planteada por divisiones...

Aquí se interrumpe el ejercicio, en aras de continuar con el desarrollo de la exposición. Por esta razón, se indica con puntos suspensivos, los cuales deberían ser utilizados con mayor frecuencia, dadas las implicaciones de innovación, nuevos paradigmas, transformaciones, adaptación, irreversibilidad de los sistemas.

El método de reflexión todo-parte permitió llegar a la identificación de las relaciones entre conocimientos, entre elementos de los sistemas y aporta fundamentalmente a la investigación.

\section{Los siete saberes}

Como eje de integración, los saberes necesarios planteados por Edgar Morin (1999) permiten relacionar el entorno con el individuo, en este caso, la organización con su entorno. Cada uno de los saberes hace posible tomar conciencia sobre las limitaciones del ser humano para concebir e interpretar adecuadamente no solo el objeto de estudio, sino su relación con el sujeto y las influencias entre uno y otro. Se presenta a continuación un esquema relacional conceptual o mentefacto que indica las relaciones entre dichos conceptos; posteriormente se hace una breve descripción de cada saber, según la figura 3.

Morin entiende por ceguera del conocimiento, la posibilidad de equívocos en la conceptualización y aplicación del conocimiento. Es la mirada parcial, incompleta, a un fenómeno 0 a las conclusiones que de esa observación se obtengan; hay errores, ilusiones, falta de memoria, paradigmas que son fuente de la ceguera. 
Figura 3. Mentefacto siete saberes

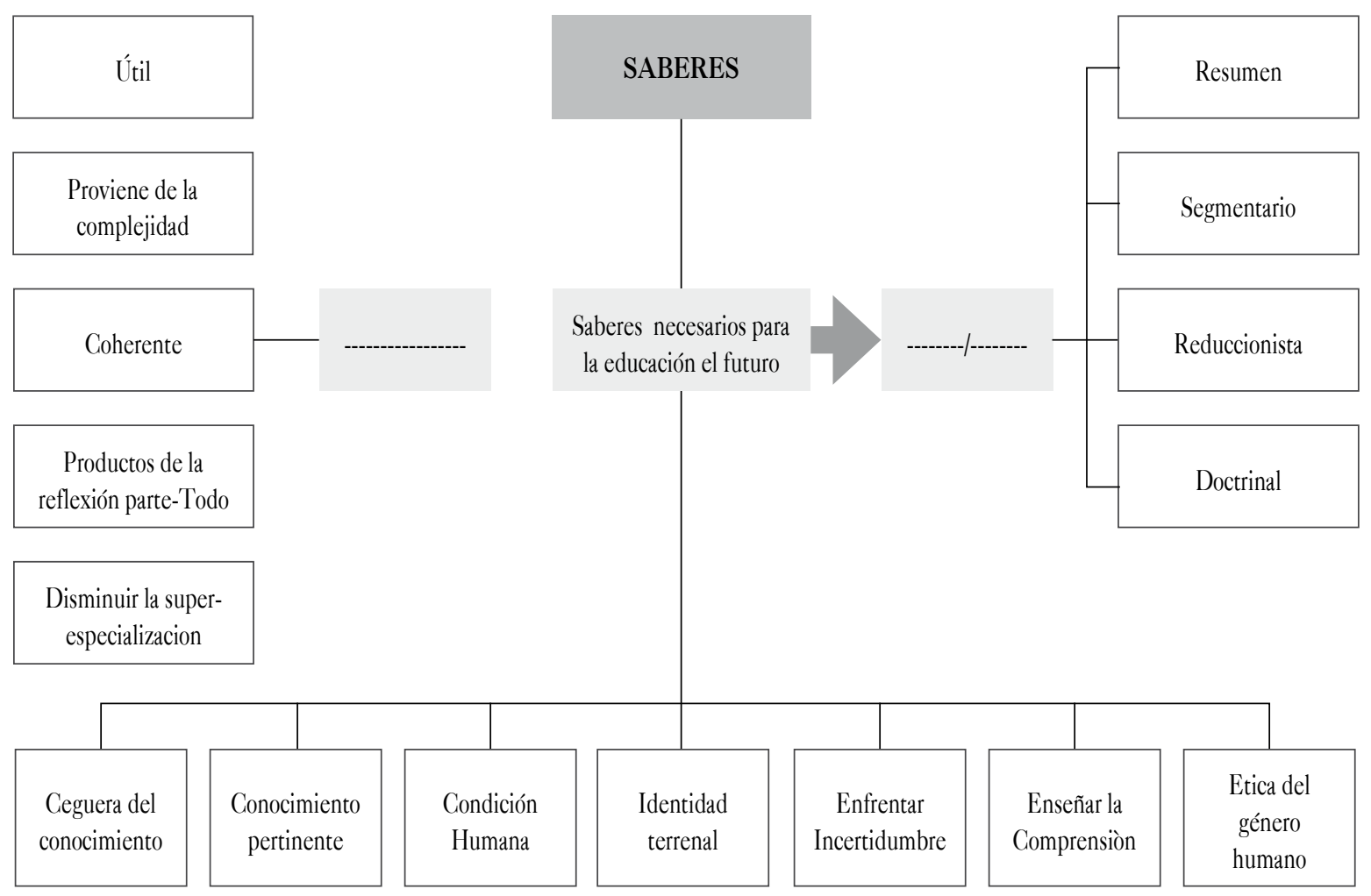

Fuente: construcción propia con base en Morin y De Zubiría (Ibáñez, 2007).

Morin relaciona el conocimiento pertinente en el ejercicio reflexivo del todo y la parte, con la identificación de las relaciones entre los elementos que constituyen un sistema. En el tema de la investigación es fundamental hacer la relación de las interacciones que afectan el sistema organizacional, del cual forman parte los sistemas de gestión, y de aquellas interacciones con el entorno que el sistema organizacional afecta.

La condición humana no debe escapar al estudio de los sistemas de gestión. De allí se obtienen características particulares, dada nuestra condición humana. Afectamos con nuestros actos a otros entornos.
Contagiamos con virus cuando nos desplazamos a otros lugares, y otros que vienen traen consigo impactos.

La identidad terrenal nos invita a reconocernos como parte de la tierra, no como usuarios simples de los recursos, sino como transformadores y, a la vez, transformados por la naturaleza, por el entorno. Las organizaciones utilizan los recursos del planeta para satisfacer necesidades.

Enfrentar la incertidumbre es una habilidad que debe ser desarrollada y aplicada para que el actuar sea preventivo, antes que correctivo, pero que se puedan tomar decisiones en medio de un nivel conocido de incertidumbre. 
En un mundo globalizado, la comprensión —en particular la humana, antes que la intelectual-, es requerida para comprender las motivaciones, las intenciones y los actos de los otros, y facilita la búsqueda de los objetivos comunes que persiguen las organizaciones y que se cristalizan en los objetivos del sistema integrado.

La ética del género humano parte de la premisa de que nadie debería ser tratado como enemigo; por el contrario, la solidaridad debe ser parte de los actos en las interacciones sociales diarias.

Si se incorporan estos saberes a la cultura organizacional, no solo se acerca a una organización caórdica, sino que aumenta la probabilidad de cumplir los objetivos, también a orientarse por los requisitos guía de las siete normas sobre sistemas de gestión analizadas en la investigación. Los conocimientos aquí esbozados permiten al investigador hacer la reflexión sobre limitaciones que se deben tener en cuenta en el desarrollo, con el fin de obtener una mejor compresión del fenómeno o la realidad estudiados.

\section{Modelo de gestión integral}

Edgar Morin (1984) caracteriza un sistema mediante el diagrama tetrárquico: desorden, organización, orden e interacción. Esta investigación, a partir de la presentación conceptual de sistema, identifica las organizaciones como sistemas sociales particulares, por lo tanto, las características del sistema les son aplicables y por ello es posible proponer la homologación sistema $=$ organización. A partir de esta identidad, se construye el modelo para la integralidad de la gestión que se presenta a continuación.

- Del desorden. El modelo construido hace corresponder e identifica un primer proceso o elemento del sistema organización que actúe como vigía, tanto de los acontecimientos y cambios externos como de los internos, en el desarrollo de las interacciones propias del sistema. Aporta datos e información.

- De la organización. En un segundo momento o proceso, el modelo indica le a la organización que adelante actividades propias del establecimiento de prioridades contando con la información del proceso anterior. Por lo tanto, en esta instancia debe determinar la necesidad de cambios si se requieren. Así, el flujo de información será constante y la valoración del impacto sobre los resultados no deberá esperar a ciclos de análisis o seguimiento a la información para la toma de decisiones. En la medida en que estos dos procesos se comuniquen adecuadamente, proporcionarán el norte inmediato a la organización. No se requieren conocimientos especializados para la captura de los datos relevantes ni para su procesamiento y evaluación frente a los objetivos.

- Del orden. El siguiente proceso hace corresponder con el modelo, los temas relacionados con el orden, la adopción y asignación de los recursos y los controles necesarios para garantizar las prioridades señaladas en el proceso anterior. Este proceso equivale, en gran medida, a la planificación conocida como la identificación de los objetivos y los recursos para lograrlos.

- De la interacción. Finalmente, el modelo hace corresponder las actividades operacionales con la interacción. Es el ciclo de la operación que consiste en el desarrollo de las interacciones con el entorno, con los elementos internos. Es la puesta en marcha del sistema.

En la parte inferior de la figura 4 se muestra una semejanza con los conceptos propuestos por Morin: de desorden: vigilancia al entorno e interna; de organización: priorización; de orden: adopción, planificación, determinación y/o asignación; y de interacción: operación. 
Figura 4. Modelo de gestión para la integralidad

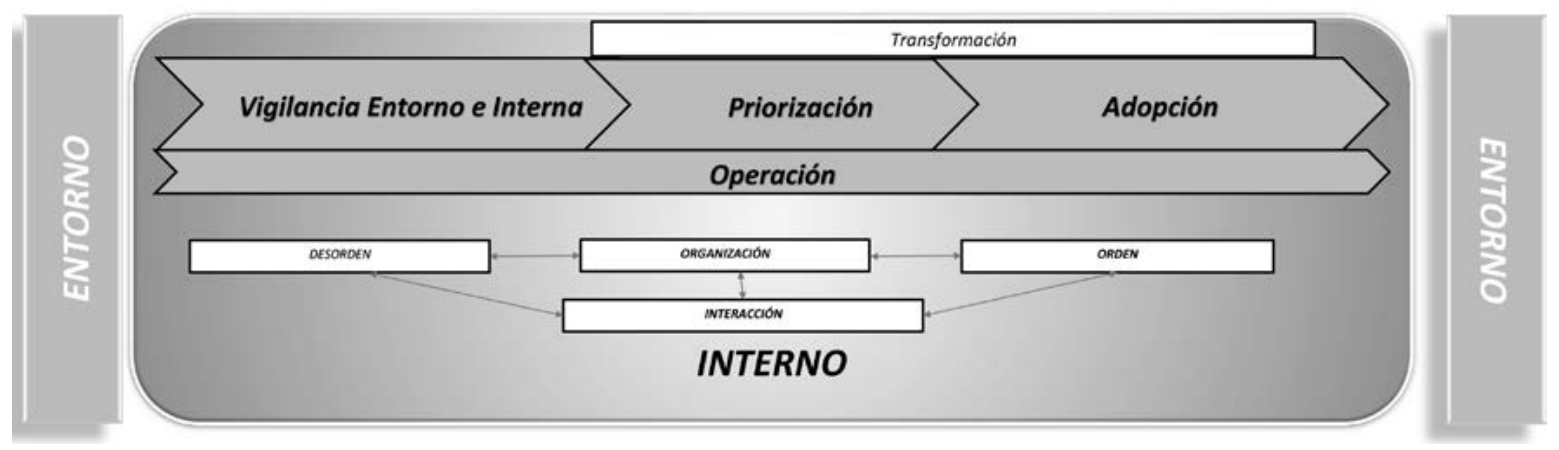

Fuente: construcción propia.

\section{Investigación aplicada}

Los resultados obtenidos con la metodología aplicada en la organización seleccionada para llevar a cabo la validación del modelo generaron un plan de acción para los aspectos relevantes, tanto los orientados a los riesgos como a las oportunidades. En cumplimiento del primer proceso, es decir vigilancia del entorno e interna, se determinaron primero las partes interesadas del proceso evaluado, y luego las especificaciones de los productos o servicios entregados a esas partes interesadas, de tal forma que fue posible identificar los riesgos y los procesos involucrados. Posteriormente, con ayuda del instrumento de priorización, se calificaron la probabilidad y el impacto, se relacionaron con los siete modelos de gestión y se construyó el plan de acción.

Más adelante se evaluó el procedimiento operacional utilizado en la organización bajo estudio; al respecto, se encontró que muy pocas de sus instrucciones contienen referencia explícita a los requisitos aplicables de cada uno de los siete modelos, de lo anterior se deduce que no se ha considerado su inclusión en dicho procedimiento. Mediante la evaluación del procedimiento fue posible determinar la afectación de este en la cultura y de la cultura sobre el procedimiento. Para afectar la cultura conscientemente, un instrumento que lo facilita es el procedimiento, siempre que contenga instrucciones específicas de los comportamientos esperados, para que ellos, en ejercicio, lo conviertan en cultura organizacional.

\section{Encuesta}

Realizada la encuesta a los diferentes niveles de la organización, se obtuvo el nivel de avance en la aplicación de cada modelo de gestión. Los resultados que muestra la figura 5 indican que los modelos de gestión que sobresalen en su nivel de implementación son los de calidad, MECI y riesgos. En términos generales, la percepción de las personas con relación a la interiorización de los modelos en el quehacer diario no se ha logrado plenamente. La información complementaria del equipo intervenido aporta que el procedimiento no incluye instrucciones sobre el comportamiento esperado que dé respuesta al enfoque con que deben realizarse las actividades, de tal forma que no solo se logre ejecutar la acción, sino que se tengan en cuenta los lineamientos de cada modelo de gestión en el momento de la ejecución de dicha acción. 
Figura 5. Resultados encuesta. Avance implementación

GENERAL
\begin{tabular}{|l|r|r|r|r|r|r|r|}
\hline & \multicolumn{1}{|c|}{$\mathbf{Q}$} & \multicolumn{1}{c|}{ Amb } & \multicolumn{1}{c|}{ SST } & \multicolumn{1}{c|}{ MECI } & \multicolumn{1}{l|}{ R } & \multicolumn{1}{c|}{ RS } & \multicolumn{1}{c|}{ SI } \\
\hline Prom & 3.7 & 1.7 & 2.2 & 3.5 & 3.9 & 2.1 & 2.5 \\
\hline
\end{tabular}

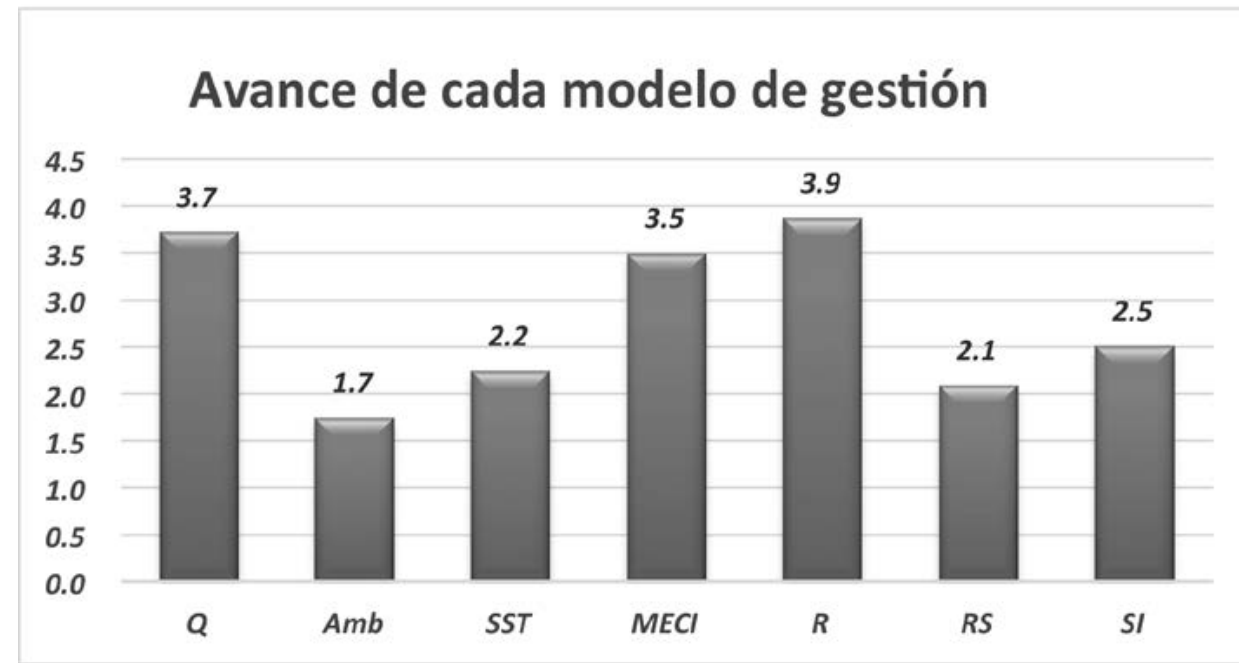

Fuente: archivo de trabajo.

Catorce profesionales participaron en el taller y evaluaron tanto su propio avance (autoevaluación) como la evaluación del procedimiento. La encuesta muestra que los responsables de procesos estiman conveniente la implementación de los siete modelos de gestión. Aumentaría $20 \%$ la probabilidad de lograr los objetivos, pasando de $62,2 \%$ a $82,2 \%$. Con este resultado se confirma la hipótesis en cuanto a que la implementación de la integralidad de la gestión de la metodología aumentaría la probabilidad de cumplir los objetivos de la empresa. Igualmente, los resultados manifiestan que la metodología utilizada en el taller, en la implementación de la metodología para la integralidad de la gestión, es adecuada y no requiere modificaciones. $\mathrm{La}$ organización, aunque no cuenta con una metodología para lograr la integración de los modelos de gestión según las respuestas (cero respuestas de 14 encuestados), tiene algún grado de avance en todos los modelos de gestión analizados, indicando que ciertas actividades son compartidas por los modelos de gestión.

\section{CONCLUSIONES}

La transdisciplinariedad como metodología puede ser adaptada a un modelo para la integralidad de la gestión, partiendo de la similitud entre un sistema, visto desde la complejidad propuesta por Edgar Morin, y una organización, concebida como sistema social. Es decir, las organizaciones pueden ser vistas como sistemas, cuyo principal propósito de gestión lo constituyan los objetivos organizacionales, con un eje integrador que es la metodología para la integralidad, en una única gestión que tenga en cuenta la incidencia de los factores que 
el entorno y su condición interna le favorezcan para el propósito de su sostenibilidad.

Son factibles la identificación y el funcionamiento de los sistemas de gestión como parte de los sistemas sociales, en particular de los sistemas organizacionales en los que se aplica la cultura organizacional, mediante una metodología que facilite a las empresas la aplicación del paradigma de la complejidad sin incurrir en complicaciones ni en dificultades operativas; esta metodología es conveniente siempre que los lineamientos de la organización se orienten al logro supremo de la sostenibilidad.

Si bien la empresa en la cual se dio la investigación aplicada no tiene un modelo para la integralidad de la gestión, muestra avances en cada uno de los siete modelos de gestión. Con base en los resultados obtenidos, la probabilidad de conseguir los objetivos en la empresa se aumentaría en $20 \%$ si se implementaran los siete modelos de gestión mediante la metodología de la integralidad para la gestión, independientemente de contar hoy en día con certificación de la calidad NTCGP1000. Esta conclusión permite aseverar que la hipótesis se cumplió al considerar que la metodología integra los siete modelos y que tanto los encuestados como los del grupo intervenido confirman que es mayor la probabilidad y la metodología es adecuada. Igualmente, como resultado de la encuesta, en la empresa no se cuenta en la actualidad con una metodología para la integralidad de la gestión, y el taller confirmó que la propuesta metodológica para la integralidad de la agestión es conveniente.

En la presentación de resultados al grupo de la organización donde se aplicó el taller para la integralidad de la gestión, se manifestó que dicha metodología no requiere modificaciones para lograr los objetivos previstos. Por medio del uso de los instrumentos diseñados fue posible determinar el nivel de implementación de los modelos de gestión y establecer el nivel de interiorización de cada uno o de todos los modelos en la empresa donde se realizó la prueba.

La aplicación de la metodología con la aplicación al proceso demostró ser muy sencilla y efectiva al poder identificar, por medio de criterios de priorización, las actividades planificadas necesarias para optimizar los esfuerzos y recursos disponibles.

\section{REFERENCIAS}

Asociación Española de Normalización y Certificación - AENOR. (2008). Guía práctica de gestión del conocimiento. UNE 412001:2008. Madrid: AENOR.

Bertalanffy, L. (1976). Teoría general de los sistemas (Trad. J. Almela. 1.a ed. en español). México: Fondo de Cultura Económica. Recuperado de goo.gl/ 050fha

British Standard Institution - BSI. (2012). Specification of common management systems requirements as a framework for intergration. Publicly Available Specification PAS 99:2008. Londres: BSI.

Cubas, J. (2010). Aportes teóricos: Migración del concepto de crisis de Edgar Morin. En C. Navajas y D. Iturriaga (coords.), Novísima. Actas del II Congreso Internacional de Historias de Nuestro Tiempo (pp. 139-145). Logroño: Universidad de La Rioja. Recuperado de goo.gl/8guiqc

Departamento Administrativo de la Función Pública DAFP. (2014). Manual técnico del modelo estándar de control interno para el Estado colombiano. Bogotá: DAFP.

Ibáñez, M. (2006). Mentefactos conceptuales como estrategia didáctico-pedagógica de los conceptos 
básicos de la teoría de muestreo aplicados en investigación en salud. Revista Ciencias de la Salud, 4 (Esp.), 62-72. Recuperado de http://www.redalyc. org/articulo.oa?id=56209908

Instituto Colombiano de Normas Técnicas y Certificación - ICONTEC. (2009). Sistemas de gestión de la calidad: Fundamentos y vocabulario. NTC - ISO 9000. Bogotá: ICONTEC.

(2008). Sistemas de gestión de la calidad. Directrices para la mejora del desempeño. NTC - ISO 9004. Bogotá: ICONTEC.

(2008). Sistemas de gestión de la calidad. Requisitos. NTC - ISO 9001. Bogotá: ICONTEC.

(2007). Sistemas de gestión en seguridad y salud ocupacional. Requisitos. NTC-OHSAS 18001. Bogotá: ICONTEC y Consejo Colombiano de Seguridad. Recuperado de goo.gl/KUsS6w

(2004). Sistemas de gestión ambiental. Requisitos con orientación para su uso. NTC-ISO 14001. Bogotá: ICONTEC. Recuperado de goo.gl/pAVuf1

(2004). Sistemas de gestión ambiental. Directrices generales sobre principios, sistemas y técnicas de apoyo. NTC-ISO14004. Bogotá: ICONTEC.

(2011). Gestión del riesgo: principios y directrices. NTC-ISO 31000. Bogotá: ICONTEC. Recuperado de g0o.gl/BV1MeZ
(2012). Directrices para la auditoría de sistemas de gestión. NTC-ISO 19011. Bogotá: ICONTEC.

(2010) Guía de responsabilidad social. GTC-ISO 26000. Bogotá: ICONTEC.

(2008). Sistema de gestión de la seguridad de la información. Requisitos NTC-ISO 27001. Bogotá: ICONTEC.

Morin, E. (1999). Los siete saberes necesarios para la educación del futuro (Trad. M. Vallejo). París: UNESCO. Recuperado de goo.gl/MNmhna . (1984). Ciencia con conciencia (Trad. A. Sánchez). Barcelona: Anthropos. . (1993). El Método. Naturaleะa de la naturaleæa. Madrid: Edic. Cátedra

Oviedo, H. y Campo-Arias, A. (2005). Aproximación al uso del coeficiente alfa de Cronbach. Revista Colombiana de Psiquiatría, 34(4), 572-580. Recuperado de http://www.redalyc.org/articulo. oa?id=80634409

Pérez, R. (1990). ¿Existe el método científico? México: El Colegio Nacional y Fondo de Cultura Económica.

Rodríguez, L.y Aguirre, J. (2011). Teorías de la complejidad y ciencias sociales. Nuevas estrategias epistemológicas y metodológicas. Nómadas. Revista Crítica de Ciencias Sociales y Jurídicas, 30(2). Recuperado de goo.gl/mxr8TT

Schvarstein, L. (2003). La inteligencia social de las organizaciones. Buenos Aires: Paidós Ibérica. 
Soracipa, N. (2009). Definición de estrategias de transformación de la cultura organizacional en función de un sistema de gestión de calidad. Revista Signos, 1(1), 139-154. DOI: 10.15332/ s2145-1389.2009.0001.09

Universidad Santo Tomás. (2004). Proyecto Educativo Institucional - PEI. Bogotá. Ediciones USTA.
Recuperado de http://www.ustamed.edu.co/ Documentos/PEI\%20Universidad\%20Santo\%20 Tomas.pdf

Vygotsky, L. (1986). Su concepción del aprendizaje y la enseñanะa. Recuperado de http://www.sld.cu/ galerias/pdf/sitios/rehabilitaciontemprana/articulo._vigostki.pdf 